- This paper demonstrates a low rate of tooth loss in a general dental practice over a period of 12 years where patients received regular dental care and careful periodontal management.

- If 'at risk' patients are identified at initial examination and appropriate treatment provided, the progression of periodontal disease in such a practice will be low over a 12-year period.

\title{
Periodontal disease incidence, progression and rate of tooth loss in a general dental practice: The results of a 12-year retrospective analysis of patient's clinical records
}

\author{
C. Nicholls ${ }^{1}$
}

One of the main objectives of a general dental practice is to teach its patients how to reduce their oral bacterial load. In other words, by teaching good oral hygiene habits, it is possible to reduce the number of bacteria and hence the number of species of bacteria present in each patient's mouth. Thus by achieving a high level of oral hygiene, the dental team hope to reduce each patient's future risk of periodontal problems, and dental caries.

In those individuals diagnosed as being susceptible to periodontal disease, a further objective must be to arrest destruction caused by the inflammation of the periodontal tissues that results from the disease. Only by achieving these objectives can the goal of maintaining a functioning dentition for the lifetime of the patient, even for those who are affected by periodontitis, ever hope to be realised.

Previously published retrospective studies ${ }^{1-4}$ have shown that periodontal treatment followed by a supportive periodontal care programme (SPC) to be very effective in controlling periodontal disease in most individuals for many years.

Some of the tools used by periodontists to measure the success of the treatment provided include methods of measuring both the incidence and the progression of periodontal disease. They include measurements such as tooth loss, bleeding on probing, changes in probing depths, changes in

1"Specialist in Periodontics, General Dental Practitioner, Charminster House Dental Practice, 65 Wellington Road, Bournemouth, Dorset BH8 8JL

${ }^{*}$ Correspondence to: Clive Nicholls

E-mail:CNichofam@aol.com

\section{Refereed Paper}

Received 24.10.02; Accepted 29.01.03

๑) British Dental Journal 2003; 194: 485-488 attachment levels and changes in the alveolar bone support around individual teeth - these measures are known as endpoints. ${ }^{5}$ Endpoints are either true or surrogate. In periodontal disease the only true endpoint is the number of teeth lost as a result of the disease.

A clinical surrogate endpoint is usually a measure of the disease process, and in periodontal disease examples are measurements of an increase or decrease of probing depth, gain or loss of periodontal attachment and gain or loss of bone around the affected teeth.

The patients in many previously reported studies are all described as having moderate to severe periodontitis, whereas in this report they make up only a proportion of the total number of patients followed over the 12 years.

It is now widely believed that the use of tooth loss as a measure of the long-term effectiveness of periodontal therapy should include all the teeth lost, including those extracted during the active phase of treatment. This is because if many of the periodontally compromised teeth are extracted during this first active phase of therapy, then there will likely be a decrease of the incidence of tooth loss during the SPC. Conversely, if severely compromised teeth are maintained through the active phase, there will likely be an increase in tooth loss during the subsequent SPC.

In many of the previously reported studies, teeth that were extracted during initial active therapy were excluded from the statistics. Where teeth lost during this first phase of treatment were not recorded, the papers were designed to show the effectiveness of treatment once the teeth that had been deemed untreatable by the providers had first been removed. In order to ascertain the actual tooth mortality in a population of patients over time, both teeth lost during initial treatment or therapy and those lost during the SPC must be known if any comparison is to be made with a population of patients where no treatment is provided.

All of the teeth that were extracted, from the moment the patient was first accepted for treatment, were included in this report. Only one previously published retrospective paper ${ }^{6}$ included those teeth also lost during initial therapy, and they found that 54\% of the total number of teeth lost during their study period were extracted during this initial phase of the treatment.

Many factors contribute to the decision to extract a tooth, including: disease 
severity at individual tooth sites, the strategic value of a tooth, aesthetics and the cost benefit ratio of available treatment options. Furthermore, teeth may need to be extracted during periodontal therapy for reasons unrelated to periodontal disease, such as unrestorable caries, recalcitrant endodontic problems and technical reconstructive problems. Evaluating the reason for tooth loss during this present review of patient's clinical notes has not been addressed, as the decision to extract a tooth is often multifactorial.

\section{AIMS}

The main aims of this report can be summarized as being:

1. To determine the incidence of patients with mild to moderate periodontitis and those with advanced periodontitis in a general dental practice.

2. To evaluate the success of treatment over a 12-year period using tooth loss as the true endpoint.

3. To record the incidence of the occurrence of new disease and progressing disease sites, by recording where teeth had probing depths increasing to $7 \mathrm{~mm}$ or greater over 12 years.

This was achieved by using both the surrogate endpoint of probing depth change over time, and the true endpoint of tooth loss both during initial active treatment, and tooth loss during the SPC. All of the initial measurements of probing depths, gingival margins, furcation involvement and tooth mobility were carried out by the author, as were all subsequent measurements throughout the 12-year audit period. A standard Williams probe was used in all measurements of probing depth and attachment loss. Finally, all the patients were seen under private contract.

\section{DEFINING THE STUDY POPULATION}

In the 6 months between February and August 1997, a consecutive record was made of all the patients who presented for recall that had first attended the practice between September 1985 and September 1986. The patients were therefore randomly selected excepting that they were regular attenders to the practice over the 12-year study period. This 1-year period 1985-86 was chosen to define the patients admitted into the study was actually the first year of opening of the practice in Bournemouth. The practice at the end of 1986 had a total of 1,200 patients.

The 156 enrolled patients were all Caucasian, and mainly from a middle economic group. They were generally well

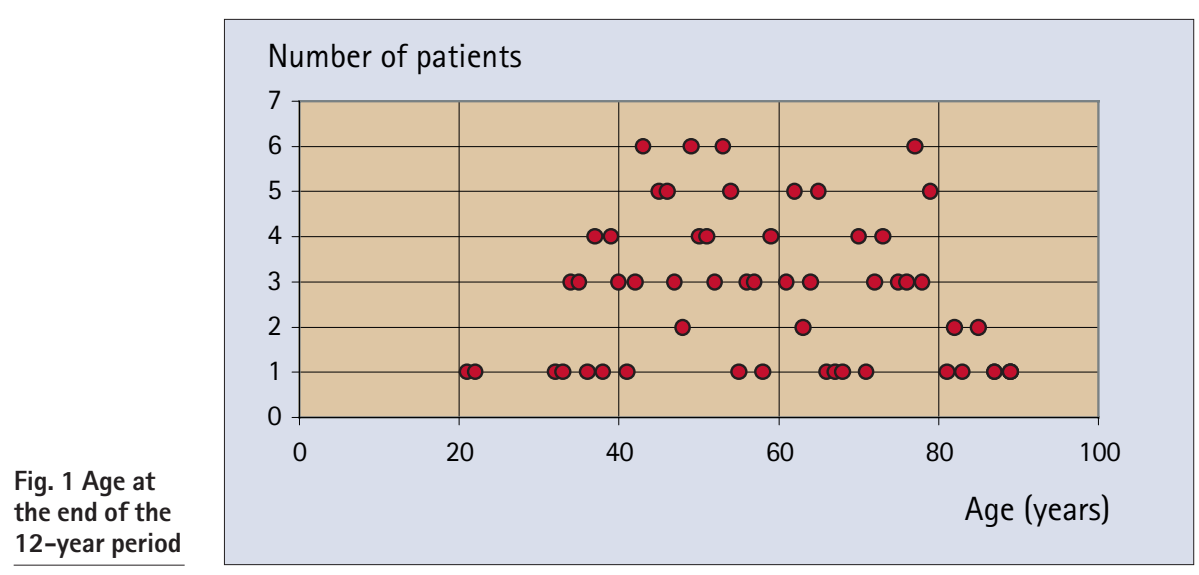

motivated in their personal and professional care and were regular attendees of the dental practice. There were 91 females and 65 males. There were 6 children who at initial examination had only few permanent teeth.

At the time of the final examination the average age was 47 . The distribution of patients according to age at the end of the study can be seen in Figure 1. They ranged from 21 to 89 years of age.

\section{ABOUT THE PRACTICE}

The practice (belonging to the author) was a general dental practice between 1985 and 1997, employing two part time hygienists, two dental nurses and a receptionist. An associate dentist also worked there between 1988 and 1997, but all of the patients in this report were on my patient list, only seeing the associate in an emergency, or while I was away.

At initial presentation patients were given a full dental examination, including a 6-point periodontal probing chart of each standing permanent tooth. Any depth of $5 \mathrm{~mm}$ or greater was recorded. Gingival margins and tooth mobilities were also charted, as were any furcation involvements of the posterior teeth. Disease progression was monitored over 12 years using the surrogate endpoint of probing depth change over time. The true endpoint of tooth loss both during initial active treatment, and tooth loss during the SPC was also recorded. All of the initial measurements of probing depths, gingival margins, furcation involvement and tooth mobility were carried out by me, as were all subsequent measurements throughout the 12-year audit period.

All patients also received their restorative dental care from myself. The root canal fillings and most other restorative treatments that were needed during the study period were provided under rubber dam. Root canals were obturated using lateral condensation gutta percha and sealed using AH26 cement.

\section{PERIODONTAL THERAPY}

\section{Initial active treatment}

Where indicated, patients with moderate to severe periodontal problems at the initial examination were prescribed a course of at least three visits to the hygienist followed 2 months later with a periodontal review with me. At this review appointment, a further 6-point probing depth examination of each standing tooth was carried out, and the effectiveness of the initial hygiene therapy and the ability of the patient to perform adequate oral hygiene evaluated.

If any probing depths of $5 \mathrm{~mm}$ or greater were still present at this review appointment, and oral hygiene was at a high standard, patients were further treated with either open or closed root planing at those specific sites. This root planing was carried out by me under local anaesthesia. If probing depths of $5 \mathrm{~mm}$ or greater were not improved, and dental hygiene was not of a sufficiently high standard, the patient was referred back to the hygienist for further non-surgical therapy. They were then kept under review until such time as their oral hygiene had improved sufficiently for further root planing to be carried out at these sites.

\section{Supportive periodontal care programme} Patients who were diagnosed as having even mild periodontitis were seen by the practice hygienists on a 3-monthly basis. The appointments were 30 minutes in duration, and involved scaling of the teeth followed by prophylaxis with polishing paste, and oral hygiene instruction and motivation. Smoking advice was also given to those that needed it, but the number of patients who smoked was sadly not recorded in the records. Over a period of 12 years periodontal health was regularly monitored by full mouth periodontal probing depth charting, and conventional dental and hygiene treatment provided as necessary. If an area of increased probing depth was discovered around a tooth during these 
examinations, and oral hygiene was maintained at a high standard, further open or closed root planing was carried out at the specific site.

\section{RESULTS REPORTED ON A PATIENT BASIS}

Of the 156 patients in the study, the patient who had their initial examination closest to the cut off date of 31.12 .86 was first seen on 2.12.86. The study period for each patient was 12 years from the date of their first examination. In all, 61 patients lost teeth during the study, and the distribution of tooth loss by patients can be seen in Figure 2.

Ninety patients (58\%) had probing depths of $5 \mathrm{~mm}$ or greater at initial examination. Thirty-three of these 90 patients (21\%) had probing depths of $7 \mathrm{~mm}$ or greater at initial examination. Of the 67 patients (43\%) who originally presented with no significant probing depths, 6 patients (9\%) went on over the 12-year period to develop at least one probing depth of $7 \mathrm{~mm}$ or greater. Eight teeth in total were so affected. Of the 90 patients (57\%) who had greater than $5 \mathrm{~mm}$ probing depths affecting at least one of their teeth at initial examination, 13 (14\%) went on to develop at least one new probing depth of $7 \mathrm{~mm}$ or greater over the 12-year period.

\section{DISCUSSION}

Teeth are extracted for a variety of reasons, not all of which are clinical. A patient may elect to have a tooth extracted for financial reasons, or a dentist may elect to extract a tooth that he may actually have been able to save for the sake of an overall treatment plan. Tooth loss remains however the only true endpoint in dentistry, and is an unequivocal measure, not prone to bias as compared to surrogate measurements like probing depths. A dentist may, for instance, probe more firmly before treatment than he does after he has completed periodontal therapy, whereas the presence or absence of a tooth is a measure that cannot be manipulated.

In Hirschfeld and Wassermann's paper, ${ }^{1}$ 600 patients were maintained over an average of 22 years in a specialist periodontal practice. After completion of initial treatment, $8.3 \%$ of teeth were lost. Teeth present when patients were first examined, but removed during this initial treatment phase were unfortunately not recorded.

Similar findings were reported by McLeod and colleagues. ${ }^{2}$ Tooth loss in these reports is used as a measure of the success of the treatment planning provided by the clinicians, rather than a measure of the actual tooth loss experienced by the patients enrolled in the studies.

All patients in these previously men-

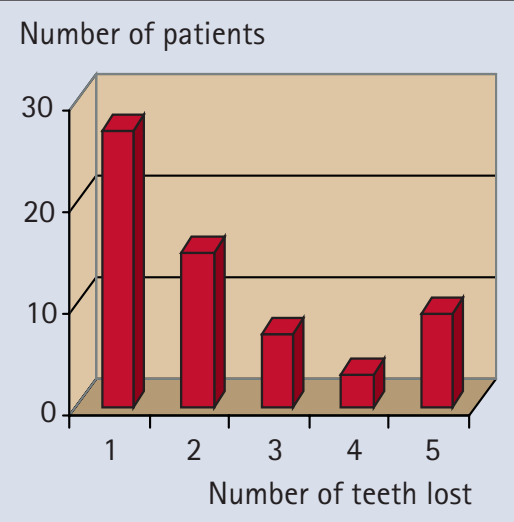

Fig. 2 Distribution of tooth loss by patient

tioned studies were described as having moderate to severe periodontitis, whereas in this present review, they only make up a proportion of the total patients that were followed. The patients in this retrospective analysis of case records also received conventional dental care, including root canal therapy where required, and restoration of teeth. Direct comparison with the results of these previously published reports should therefore only be made with great care, appreciating the differences in the populations studied, and the exclusion of teeth lost during active treatment.

Despite these differences in design, if any comparison is to be made, then it is best made using tooth mortality rates. This is calculated by dividing the number of teeth lost by the number of patients, then dividing this number again by the number of years over which the audit was conducted. Hirschfield and Wasserman were able to achieve a tooth mortality rate of 0.08 , the same finding as in the present study over its 12-year period of investigation. In other words, 0.08 teeth were lost per person per year of the study.

A comparison with the paper by Tonetti et al., which was a 6-year retrospective study but which recorded teeth lost in the initial active phase of treatment can also be made using tooth mortality rates. In this paper the tooth mortality rate was 0.35. Fifty-four per cent of teeth extracted were lost during active therapy in the Tonetti paper as compared with the current reports findings where only 32\% of the teeth lost in the first 6 years were removed during this same initial treatment period.

It must again be remembered that not all the patients in the present investigation presented with periodontal disease. Direct comparison of the two papers is further complicated by the fact that the Tonetti paper is only an average 6-year study period, patients being enrolled for as little as 5 months, and as long as 278 months. It is difficult, therefore, to directly compare the data with the actual 12-year longitudinal data of the present investigation.

Although attachment levels were recorded in the study population of this report, changes in attachment level were inadequately recorded, and are not therefore reported.

When compared with Buckley and Crowley's ${ }^{7}$ result of a tooth mortality rate of 0.25 in patients that were untreated for periodontal disease over a 10 -year period, treatment followed by an SPC would seem to decrease the risk of tooth loss. Comparisons between such controlled and uncontrolled patient groups in retrospective audits may not be scientifically astute. Kocher's paper $^{8}$ however would suggest that only active treatment followed by an SPC prevents further tooth loss as a result of periodontal disease.

Patients at risk of periodontal problems would appear to be quite a high proportion of the total number of patients seen in a general practice from the data published in this audit. In a hypothetical average dental practice of 1,200 regularly-attending middle class adult patients, 684 patients could be expected to have at least one tooth with a probing depth of 5-6 mm, 252 could be expected to have at least one tooth with a probing depth of $7 \mathrm{~mm}$ or greater.

Progression of periodontal disease over the 12-year period would appear to be low in a well-controlled patient population. In the same hypothetical practice of 1,200 patients, of those patients that originally presented with no probing depths of $5 \mathrm{~mm}$ or greater, 4 patients every year could be expected to develop at least one probing depth of $7 \mathrm{~mm}$ or greater. Of those who originally presented with at least one tooth with a $5 \mathrm{~mm}$ probing depth, 8 patients each year could be expected to develop at least one new site of $7 \mathrm{~mm}$ or greater. In other words, $1 \%$ of patients exhibited a progressing disease site each year.

One disadvantage of this paper's retrospective analysis is that it does not address what happened to the patients who did not attend 12 years later in the 6-month period between February and August 1997. Determining whether these patients had just delayed examination and not attended during the audited period, or had moved from the area, become dissatisfied with the care they received from the practice or perhaps even died, is beyond the scope of this audit. However, this does leave the study open to criticism in that the population may demonstrate bias towards patients satisfied with the care that they received, as compared with those who were not happy.

Another criticism of this paper's design would be the changes in treatment options available to a general dental practitioner since 1985-86. Periodontal 
treatment has not changed significantly, but the introduction of reliable dental implants may well mean that more teeth will be extracted in future, in order to make way for a more satisfactory dental solution using osseo-integrated implants. Where lower incisors are the only remaining teeth, they might be sacrificed today for the sake of such a reconstruction, where they would have been maintained in 1985-86 and a lower partial denture used to restore the posterior premolar occlusion. Dental implants were only introduced into my practice as a treatment option in 1994.

Finally, because the number of extracted teeth is quite small, the significance of some of the findings of this paper might also be called into question. The length of time over which the analysis was carried out must however be considered when evaluating its significance. Current fashion in evidence-based research gives more value to progressive rather than retrospective reporting, because of the inevitable bias that occurs in reporting on patients retrospectively, as I have already acknowledged.

The problem with prospective reporting is that funding for a 12-year study would prove difficult to obtain, and does not anyway remove the issue of bias from the data. I cannot fail but observe that in much of the current published literature, not many of the research workers are prepared to publish negative results, skewing published research because of their desire to demonstrate significance and value.

In a profession where so much knowledge is based upon clinical experience, and techniques handed down from teacher to pupil, the importance of longitudinal retrospective analysis might currently be being undervalued by the scientific community.
1. Hirshfield L. Wasserman B. A long term survey of tooth loss in 600 treated periodontal patients. J Periodontol 1978; 49: 225-237.

2. McLeod D E, Lainson PA, Spivey J D. The effectiveness of periodontal treatment as measured by tooth loss. J Am Dent Assoc 1997; 128: 316-24.

3. Nevins M. Long-term periodontal maintenance in private practice. [Review] [14 references]. J Periodonto/ 1996; 23: 273-277.

4. Nicholls C. A 12-year retrospective audit study of tooth loss in a general dental practice. Br Dent $J 2000$ 189: 98-99

5. Hugoel P P, DeRouen TA. A survey of endpoint characteristics in periodontal clinical trials published 1988-1992, and implications for future studies. J Periodontol 1995; 22: 397-407.

6. Tonetti M S, Steffen P, Muller Campanile V, Suvan J, Lang N P. Initial extractions and tooth loss during supportive care in a periodontal population seeking comprehensive care. J Periodonto/ 2000; 27: 824-831.

7. Buckley LA, Crowley M J. A Longitudinal Study of Untreated Periodontal Disease. J Periodontol 1984;11: 523-530.

8. Kocher T, Konig J, Dzierzon U, Sawaf H, Plagmann H C Disease progression in periodontally treated and untreated patients - a retrospective study. J Periodontol 2000; 27: 866-872.

\section{BDA Information Centre Services}

\section{Did you know?}

- As a BDA member you can gain access to one of the best dental information services in the world

- You don't have to be based in London to use the service

- You can borrow books, videos and information packages

- You can borrow up to eight items via the postal system The only cost to you is the cost of the return postage. If you're not sure what to request then telephone us and we can advise you.

- You are entitled to free MEDLINE searches Telephone us with a subject and we will send you a list of relevant references with abstracts.

- You can request photocopies of journal articles There is a small charge for this service and you need to fill in a Photocopy Request Form first. Telephone us if you would like one of these forms.

- BDA Members can view the latest Current Dental Titles on our web site free of charge.

These are Medline-based lists of references on eight areas of dentistry which are available to BDA members only on the our web site and which are updated twice yearly. Just use your password with which you have been issued.

For further details of any of these services dial: 02075634545.

\section{Contact us via e-mail at: Infocentre@bda-dentistry.org.uk}

Visit the Information Centre web pages at: http://www.bda-dentistry.org.uk 